\title{
Generalized additive models in business and economics
}

\author{
Sunil K. Sapra \\ California State University, Los Angeles, USA \\ E-mail: ssapra@calstatela.edu
}

\begin{abstract}
The paper presents applications of a class of semi-parametric models called generalized additive models (GAMs) to several business and economic datasets. Applications include analysis of wage-education relationship, brand choice, and number of trips to a doctor's office. The dependent variable may be continuous, categorical or count. These semiparametric models are flexible and robust extensions of Logit, Poisson, Negative Binomial and other generalized linear models. The GAMs are represented using penalized regression splines and are estimated by penalized regression methods. The degree of smoothness for the unknown functions in the linear predictor part of the GAM is estimated using cross validation. The GAMs allow us to build a regression surface as a sum of lower-dimensional nonparametric terms circumventing the curse of dimensionality: the slow convergence of an estimator to the true value in high dimensions. For each application studied in the paper, several GAMs are compared and the best model is selected using AIC, UBRE score, deviances, and R-sq (adjusted). The econometric techniques utilized in the paper are widely applicable to the analysis of count, binary response and duration types of data encountered in business and economics.
\end{abstract}

Keywords: Generalized additive models (GAMs), Generalized Linear Models (GLMs), Logit Models, Poisson Regression Models, Penalized Regression Splines.

\section{Introduction}

Discrete choice and count data regression models are pervasive in business and economics. Logit and Poisson regression models are the most commonly employed models for non-normal data. Common applications of Logit models include analysis of brand choice data in marketing (Baltas [1] and Guadagni and Little [4]) and transportation choice data in economics (Greene [3] and Manski and McFadden [12]). Poisson regression models have been applied in the analysis of data on patents, number of trips to a doctor's office, and number of shipping accidents. These regression models belong to the class of generalized linear models (GLMs), which relax the assumption that the response is normally distributed by allowing it to follow any distribution from the exponential family, such as normal, Poisson, binomial, gamma etc. Inference for GLMs is based on likelihood theory. McCullagh and Nelder [11] provide an authoritative account of GLMs and Cameron and Trivedi [2] and Greene [3] provide econometric applications.

In recent years, semi-parametric extensions of linear regressions have been employed in business and economics. Nevertheless, there have been relatively few applications of semi-parametric extensions of generalized linear models in business and economics despite applications in other fields (see Hastie and Tibshirani [7]). This paper attempts to fill this gap. We present several econometric applications of the generalized additive model (GAM), a semi-parametric extension of GLMs and demonstrate the usefulness of these semiparametric models for the analysis of continuous, discrete, and count data. A GAM is a semi-parametric GLM in which part of the linear predictor is specified in terms of a sum of unknown smooth functions of explanatory variables. Generalized additive models (GAMs) are a powerful generalization of linear, logistic, and Poisson regression models. GAMs are very flexible, and can provide an elegant fit in the presence of nonlinear relationships. GAMs and GLMs can be applied in similar situations, but they serve different analytic objectives. GLMs emphasize estimation and inference for the parameters of the model, while GAMs focus on exploring data nonparametrically. The GAM approach offers more flexibility in model form than the GLM approach does. These models estimate an additive approximation to the multivariate link function. The main benefit of this approach is that each of the individual additive terms is estimated using a univariate smoother instead of a multivariate smoother for a high-dimensional non-parametric term circumventing the curse of dimensionality: the slow convergence of an estimator to the true value in high dimensions. The GAM formulation of the GLM regression models allows us to build a regression surface as a sum of lower-dimensional nonparametric terms. Two popular approaches to estimation of GAMs are backfitting with local scoring algorithm (Hastie and Tibshirani [6]) and penalized regression 
splines (Wood ([14] and [15])). Backfitting has the advantage that it can be used with any scatterplot smoother while the penalized regression splines method has the advantage that estimation of the smoothing parameter using generalized cross validation is integrated into estimation (see Wood [15]). For a discussion of other approaches to GAM estimation, see Wood [15].

This paper presents econometric applications of the GAM extensions of the generalized linear models (GLMs) including the linear regression model and demonstrates that the GAMs can overcome a serious weakness of the GLMs in failing to identify the nonlinearities in the link function. The paper is organized as follows. Section 2 introduces the generalized additive model (GAM). Section 3 presents the penalized regression splines method for the estimation of GAMs. Section 4 presents an econometric application of GAM Gaussian model to wage-education data. Section 5 presents an econometric application of GAM Logit model to Cracker data on choice between four cereal brands and Section 6 presents an application of GAM Poisson regression model to the number of trips to a doctor's office. Section 7 provides some concluding remarks.

\section{Generalized additive models}

Generalized additive models are nonparametric generalized linear models. These models extend traditional linear models by allowing for a link between the nonlinear predictor $f\left(x_{1}, \ldots, x_{p}\right)$ and the expected value of $y$. This amounts to allowing for an alternative distribution for the underlying random variation besides just the normal distribution. While Gaussian models can be used in many statistical applications, for several types of problems they are not appropriate. For example, the normal distribution may not be adequate for modeling discrete responses such as counts, or bounded responses such as proportions.

Generalized additive models consist of a random component, an additive component, and a link function relating these two components. The response $y$, the random component, is assumed to have a density in the exponential family

$f_{Y}(y ; \theta, \varphi)=\exp \left\{\frac{y \theta-b(\theta)}{a(\varphi)}+c(y . \varphi)\right\}$,

where $\theta$ is called the natural parameter and $\phi$ is the scale parameter. The normal, binomial, and Poisson distributions are all in this family. Unlike the generalized linear models, the mean $\mu=\mathrm{E}\left(y \mid x_{1}, x_{2}, \ldots, x_{p}\right)$ is not linked to the linear predictor $\sum x_{i j} \beta_{j}$, but to the nonlinear nonparametric predictor

$\eta=g(\mu)=\alpha+\sum_{j=1}^{p} s_{j}\left(x_{j}\right)$,

where $s_{l}(\cdot), \ldots, s_{p}(\cdot)$ are smooth nonparametric functions, which defines the additive component. Finally, the relationship between the mean $\mu$ of the response variable and $\eta$ is defined by a link function $g(\mu)=\eta$. The most commonly used link function is the canonical link, for which $\eta=\theta$.

A combination of backfitting and local scoring algorithms are used in the actual fitting of the model. In order to fit GAMs to the data, following Wood [15], we use basis expansions of smooth functions and penalized likelihood maximization for model estimation in which wiggly models are penalized more heavily than smooth models in a controllable manner, and degree of smoothness is chosen based on cross validation, or AIC or Mallows' criterion.

The generalized additive models are fit to count data or binary response data by maximizing a penalized log likelihood or a penalized log partial-likelihood. To maximize it, the backfitting procedure is used in conjunction with a maximum likelihood or maximum partial likelihood algorithm (Hastie and Tibshirani ([6] and [8]) and Wood [15]). The NewtonRaphson method for maximizing log-likelihoods in these models can be presented in an IRLS (iteratively reweighted least squares) form. It involves a repeated weighted linear regression of a constructed response variable on the covariates: each regression yields a new value of the parameter estimates which give a new constructed variable, and the process is iterated. In the generalized additive model, the weighted linear regression is simply replaced by a weighted backfitting algorithm (Hastie and Tibshirani [6]).

\section{Estimation of generalized additive models using Penalized regression method}

\section{Algorithm for Penalized Iteratively Re-weighted Least Squares (PIRLS) (Wood [15])}

The GAMs are fit to data by maximizing a penalized log likelihood or a penalized log partial-likelihood. The following algorithm is used to implement these methods. The R-package mgcv (Wood [14]) was used for computations. 
Step 1: Initialize $\hat{\alpha}=g\left(1 / N \sum_{i=1}^{N} y_{i}\right), s_{j}^{0}=0, j=1,2, \ldots, p$.

Step 2: Construct an adjusted dependent variable $z_{i j}$ as

$z_{i}=\eta_{i}^{0}-\left(y_{i}-\mu_{i}^{0}\right)\left(\partial \eta_{i} / \partial \mu_{i}\right)_{0}$

$\eta_{i}^{0}=g\left(\mu_{i}^{0}\right)=\alpha^{0}+\sum_{j=1}^{p} s_{j}^{0}\left(x_{i j}\right)$ and $\mu_{i}^{0}=g^{-1}\left(\eta_{i}^{0}\right)$.

Step 3: Compute weights

$w_{i}=\left(\partial \mu_{i} / \partial \eta_{i}\right)_{0}\left(V_{i}^{0}\right)^{-1}$,

where $V_{i}^{0}$ is the variance of $y$ at $\mu_{i}^{0}$,

Step 4: Penalized Spline Re gression

Minimize $\|\sqrt{W}(z-X \beta)\|^{2}+\lambda \beta^{\prime} S \beta$ with respect to $\beta$, where $X$ is the matrix of data on basis

functions used to represent the regressionfunction, $W$ is a diagonal matrix with $i$-th diagonal

element $w_{i}, S$ is a matrix of known coefficients in the penalty function $\beta^{\prime} S \beta$ and $\lambda$ is a

smoothing parameter. Compute $s_{j}^{1}, \eta^{l}$, and $\mu_{i}^{1}$, the second stage estimates of $s_{j}, \eta$, and $\mu_{i}$.

Step 5: Repeat steps 2-4 replacing $\eta^{0}$ by $\eta^{1}$ until the difference between two successive

values of $\eta$ is less than a small prespecified number and convergence is obtained.

\section{The generalized additive Gaussian model}

The generalized additive Gaussian model assumes that

$g\left(\mu_{i}\right)=\mu_{i}=\alpha+\sum_{j=1}^{p} s_{j}\left(x_{i j}\right)$ for the identity link and

$g\left(\mu_{i}\right)=\ln \mu_{i}=\alpha+\sum_{j=1}^{p} s_{j}\left(x_{i j}\right)$ for the log link.

The adjusted dependent variable $z$ and the weights $w$ used in the algorithm above are

$z_{i}=y_{i}$ and $w_{i}=1$ for all $i$ for the identity link and

$z_{i}=\ln y_{i}$ and $w_{i}=1$ for all $i$ for the log link.

The functions $s_{1}, s_{2}, \ldots, s_{p}$ are estimated by the algorithm described earlier.

\subsection{An Empirical Application of GAM Gaussian Model to data on wages}

\section{Variable Definitions and Data Description}

A small subset of CPS data on labor force status are taken are from Hill et al [9]. The dependent variable is WAGE. The variables are defined as follows.

$W A G E=$ Earnings per hour

$E D U C=$ Years of education

$E X P E R=$ Post education years experience

$H R S W K=$ Usual hours worked per week

$M A R R I E D=1$ if married.

\begin{tabular}{llllll}
\multicolumn{7}{c}{ Table 1: Summary Statistics } \\
\hline Variable & Obs & Mean & Std Dev & Min & Max \\
\hline WAGE & 1000 & 20.20122 & 12.1038 & 2.03 & 72.13 \\
EDUC & 1000 & 10.689 & 2.44013 & 1 & 16 \\
EXPER & 1000 & 26.501 & 12.99041 & 3 & 64 \\
HRSWK & 1000 & 39.24 & 11.44611 & 0 & 99 \\
\hline
\end{tabular}




\section{Models}

The following models were fitted to the data. Model 1 is a linear regression model with WAGE as the dependent variable and EDUC, EXPER, and HRSWK as the independent variables, which is a GLM model with identity link, Model 2 introduces a quadratic term, the square of HRSWK. Model 3 is a GAM model with the identity link, which introduces a nonparametric smooth term for HRSWK since a high degree of nonlinearity is observed in a partial residual plot of $H R S W K$ displayed in Fig. 1. Models 4, 5, 6, and 7 use the identity link with $\ln (W A G E)$ as the dependent variable. Model 4 is a GAM model with independent variables EDUC, EXPER, and HRSWK, which is a GLM model with identity link for $\ln (W A G E)$. Model 5 is a GAM model which employs parametric terms for EDUC and EXPER but a nonparametric smooth term for HRSWK. Model 6 is a GAM model, which includes parametric terms for EDUC and EXPER, a parametric interaction term for interaction between EXPERIENCE and MARRIED, but a nonparametric smooth term for HRSWK. Model 7 replaces the parametric interaction term for interaction between EXPERIENCE and MARRIED, with a nonparametric smooth interaction term.

Model 1: GLM Normal with Identity Link

$\eta=g(\mu)=\beta_{1}+\beta_{2} E D U C+\beta_{3} E X P E R+\beta_{4} H R S W K$

Model 2: GLM Normal with a Quadratic Term and Identity Link

$\eta=g(\mu)=\beta_{1}+\beta_{2} E D U C+\beta_{3} E X P E R+\beta_{4} H R S W K$

$+\beta_{4} H R S W K S Q$

Model 3:Generalized Additive Re gression Models with Identity Link for Wages and a

Nonparametric Smooth Term for HRSWK

$\eta=g(\mu)=\beta_{1}+\beta_{2} E D U C+\beta_{3} E X P E R+\beta_{4} H R S W K+s(H R S W K)$

Model 4: GLM Normal with Identity Link for ln(Wages)

$\eta=g(\mu)=\beta_{1}+\beta_{2} E D U C+\beta_{3} E X P E R+\beta_{4} H R S W K$

Model 5: GAM Normal with Identity Link for $\ln ($ Wages) and a Nonparametric Smooth

Term for HRSWK

$\eta=g(\mu)=\beta_{1}+\beta_{2} E D U C+\beta_{3} E X P E R+s(H R S W K)$

Model 6: GAM Normal with Identity Link for $\ln ($ Wages ), a Nonparametric Smooth Term

for HRSWK and an Interaction

$\eta=g(\mu)=\beta_{1}+\beta_{2} E D U C+\beta_{3} E X P E R+\beta_{4} E X P E R * M A R R I E D+s(H R S W K)$

Model 7: GAM Normal with Identity Link for In(Wages), a Nonparametric Interaction

Term and a Smooth Term for HRSWK

$\eta=g(\mu)=\beta_{1}+\beta_{2} E D U C+\beta_{3} E X P E R+s(E X P E R * M A R R I E D)+s(H R S W K)$

Table 2: Model 1: GLM Normal with Identity Link

\begin{tabular}{lllll}
\hline Variable & Estimate & Std. Error & t-ratio & p-value \\
\hline INTERCEPT & -14.93450 & 1.95933 & -7.622 & $5.80 \times 10^{-14^{* * *}}$ \\
EDUC & 2.14545 & 0.13904 & 15.430 & $<2 \times 10^{-16^{* * * *}}$ \\
EXPER & 0.12594 & 0.02578 & 4.886 & $1.20 \times 10^{-6^{* * *}}$ \\
HRSWK & 0.22593 & 0.02920 & 7.737 & $2.49 \times 10^{-14^{* * *}}$ \\
\hline
\end{tabular}

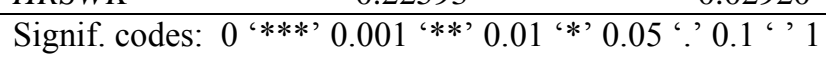

(Dispersion parameter for Gaussian family taken to be 108.5920)

Null deviance: 146356 on 999 degrees of freedom

Residual deviance: 108158 on 996 degrees of freedom

AIC: 7531.5

Number of Fisher Scoring iterations: 2

Table 3: Model 2: GLM Normal with a Quadratic term and Identity Link

\begin{tabular}{lllll}
\hline Variable & Estimate & Std. Error & t-ratio & p-value \\
\hline INTERCEPT & -18.284918 & 2.357264 & -7.757 & $2,15 \times 10^{-14^{* * *}}$ \\
EDUC & 2.155801 & 0.138724 & 15.540 & $<2 \times 10^{-16^{* * *}}$ \\
EXPER & 0.123334 & 0.025728 & 4.794 & $1.89 \times 10^{-6^{* * *}}$ \\
HRSWK & 0.423466 & 0.083016 & 5.101 & $4.04 \times 10^{-7^{* * * *}}$ \\
HRSWK^2 & -0.002659 & 0.001047 & -2.541 & $0.0112^{*}$ \\
\hline
\end{tabular}


Signif. codes: 0 ‘***’ 0.001 '**' 0.01 '*’ 0.05 ‘' 0.1 ' ' 1

(Dispersion parameter for Gaussian family taken to be 108.0003)

Null deviance: 146356 on 999 degrees of freedom

Residual deviance: 107460 on 995 degrees of freedom

AIC: 7527

Number of Fisher Scoring iterations: 2

Table 4: Model 3: GAM Normal with Identity Link

\begin{tabular}{lllll}
\hline Variable & Estimate & Std. Error & t-ratio & p-value \\
\hline INTERCEPT & -5.70533 & 1.75403 & -3.253 & $0.001118^{* *}$ \\
EDUC & 2.11854 & 0.13787 & 15.367 & $<2 \times 10^{-16}$ \\
EXPER & 0.12307 & 0.02546 & 4.833 & $1.56 \times 10^{-6}$ \\
\hline
\end{tabular}

Approximate significance of smooth terms:

\begin{tabular}{|l|l|l|l|l|}
\hline Variable & Estimated df & Refined df & F & p-value \\
\hline$s(H R S W K)$ & 4.295 & 5.284 & 16.51 & $<2 \times 10^{-16 * *}$ \\
\hline
\end{tabular}

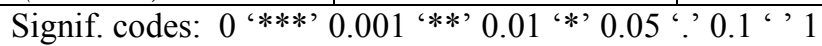

R-sq. $(\operatorname{adj})=0.278$ Deviance explained $=28.3 \%$

GCV score $=106.52$ Scale est. $=105.75 \quad \mathrm{n}=1000$

$\mathrm{AIC}=7508.195$

Null Deviance: 146355.6 on 999 degrees of freedom

Residual Deviance: 108157.6 on 996 degrees of freedom

Number of Local Scoring Iterations: 2

\section{Nonparametric exploration of nonlinearity}

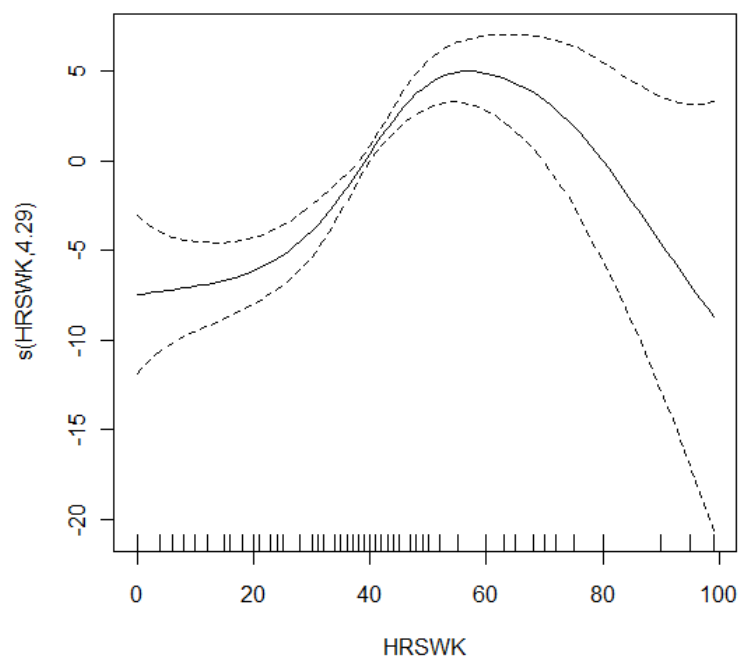

Fig. 1: Partial residuals plot of HRSWK

Table 5: Model 4: GLM Normal with Identity Link for $\ln$ (Wages)

\begin{tabular}{lllll}
\hline Variable & Estimate & Std. Error & t-ratio & p-value \\
\hline INTERCEPT & 1.260476 & 0.104063 & 12.113 & $<2 \times 10^{-16^{* * * *}}$ \\
EDUC & 0.112250 & 0.007222 & 15.544 & $<2 \times 10^{-16^{* * * *}}$ \\
EXPER & 0.005845 & 0.001250 & 4.678 & $3.30 \times 10^{-6^{* * * *}}$ \\
HRSWK & 0.008831 & 0.001358 & 6.503 & $1.25 \times 10^{-10^{* * *}}$ \\
\hline
\end{tabular}

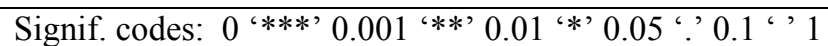

(Dispersion parameter for Gaussian family taken to be 107.7871)

Null deviance: 146356 on 999 degrees of freedom

Residual deviance: 107356 on 996 degrees of freedom

AIC: 7524.032

Number of Fisher Scoring iterations: 6 
Table 6: Model 5: GAM Normal with Identity Link for $\ln$ (Wages)

\begin{tabular}{lllll}
\hline Variable & Estimate & Std. Error & t-ratio & p-value \\
\hline INTERCEPT & 1.624786 & 0.095485 & 17.016 & $<2 \times 10^{-16^{* * * *}}$ \\
EDUC & 0.110449 & 0.007118 & 15.518 & $<2 \times 10^{-16^{* * * *}}$ \\
EXPER & 0.005555 & 0.001235 & 4.497 & $7.7 \times 10^{-6^{* * * *}}$ \\
\hline
\end{tabular}

Approximate significance of smooth terms:

\begin{tabular}{|l|l|l|l|l|}
\hline Variable & Estimated df & Refined df & F & p-value \\
\hline$s(H R S W K)$ & 3.974 & 4.961 & 14.20 & $2.33 \times 10^{-13 * *}$ \\
\hline
\end{tabular}

Signif. codes: 0 ‘***’ 0.001 '**’ 0.01 '*’ 0.05 ‘' 0.1 “ ' 1

R-sq. $(\operatorname{adj})=0.293$ Deviance explained $=29.7 \%$

GCV score $=104.37$ Scale est. $=103.64 \quad \mathrm{n}=1000$

$\mathrm{AIC}=7487.733$

Null Deviance: 146355.6 on 999 degrees of freedom

Residual Deviance: 102866.4 on 992.9999 degrees of freedom

Number of Local Scoring Iterations: 7

Table 7: Model 6: GAM Normal with an Interaction Identity Link for ln(Wages)

\begin{tabular}{lllll}
\hline Variable & Estimate & Std. Error & t-ratio & p-value \\
\hline INTERCEPT & 1.649134 & 0.095606 & 17.249 & $<2 \times 10^{-16^{* * *}}$ \\
EDUC & 0.109249 & 0.007107 & 15.373 & $<2 \times 10^{-16^{* * *}}$ \\
EXPER & 0.002676 & 0.001576 & 1.698 & 0.08985 \\
EXPR ${ }^{*}$ MARRIED & 0.003586 & 0.001172 & 3.060 & $0.00228^{* *}$ \\
\hline
\end{tabular}

Signif. codes: 0 ‘***' 0.001 '**' 0.01 '*’ 0.05 ‘' 0.1 “ ' 1

Approximate significance of smooth terms:

\begin{tabular}{|l|l|l|l|l|}
\hline Variable & Estimated df & Refined df & F & p-value \\
\hline$s(H R S W K)$ & 3.885 & 4.859 & 14.54 & $1.83 \times 10^{-13 * *}$ \\
\hline
\end{tabular}

Signif. codes: 0 ‘***' 0.001 '**' 0.01 '*’ 0.05 ' ’ 0.1 ' ' 1

R-sq. $(\operatorname{adj})=0.299$ Deviance explained $=30.4 \%$

GCV score $=103.55$ Scale est. $=102.74 \quad \mathrm{n}=1000$

AIC $=7479.896$

Null Deviance: 146355.6 on 999 degrees of freedom

Residual Deviance: 101865.4 on 991.9999 degrees of freedom

Number of Local Scoring Iterations: 7

Table 8: Model 7: GAM Normal with an Interaction term and Identity Link for $\ln$ (Wages)

\begin{tabular}{|c|c|c|c|c|}
\hline Variable & Estimate & Std. Error & t-ratio & p-value \\
\hline INTERCEPT & 1.638620 & 0.102779 & 15.943 & $<2 \times 10^{-16^{* * * *}}$ \\
\hline$E D U C$ & 0.108653 & 0.007056 & 15.399 & $<2 \times 10^{-16^{* * *}}$ \\
\hline EXPER & 0.005633 & 0.001888 & 2.983 & $0.00293 * *$ \\
\hline
\end{tabular}

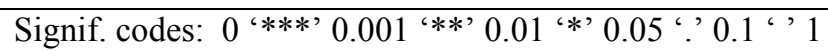

Approximate significance of smooth terms:

\begin{tabular}{|l|l|l|l|l|}
\hline Variable & Estimated df & Refined df & F & p-value \\
\hline$s($ HRSWK $)$ & 3.928 & 4.908 & 13.251 & $2.46 \times 10^{-12} * *$ \\
\hline$s($ EXPER $*$ MARRIED $)$ & 2.571 & 3.229 & 6.937 & $7.95 \times 10^{-5} * *$ \\
\hline
\end{tabular}

Signif. codes: 0 ‘***' 0.001 '**' 0.01 '*’ 0.05 '? 0.1 ' ' 1

R-sq. $(\operatorname{adj})=0.308$ Deviance explained $=31.4 \%$

GCV score $=102.28$ Scale est. $=101.31 \quad n=1000$

$\mathrm{AIC}=7467.548$

Null Deviance: 146355.6 on 999 degrees of freedom 
Residual Deviance: 100082.5 on 989 degrees of freedom

Number of Local Scoring Iterations: 6

\subsection{Comparing the Models}

Estimation results are presented in tables 2 through 8 . A comparison of models using the AIC presented in Table 9 suggests that models 6 and 7, which employ $\ln (W A G E S)$ as the response variable and allow for interaction between EXPER and MARRIED have the lowest AICs among the models considered and are therefore the best models. Model 7 , a generalized additive model for $\ln (W A G E S)$, which includes a nonparametric interaction term between EXPER and MARRIED has the lowest AIC and UBRE score among the seven models studied . Model 6, which allows parametric interaction term between EXPER and MARRIED has the second lowest AIC and UBRE score. At the other extreme, Model 1, a generalized linear Gaussian model for WAGES, has the highest AIC suggesting that it is the poorest model among the models considered.

\begin{tabular}{ll}
\multicolumn{2}{c}{ Table 9: Models and the AICs } \\
\hline MODEL & AIC \\
\hline 1 & 7531.5 \\
2 & 7527 \\
3 & 7508.195 \\
4 & 7524.032 \\
5 & 7487.733 \\
6 & 7479.896 \\
7 & 7467.548 \\
\hline
\end{tabular}

\section{The generalized additive Logit model}

The generalized additive Logit model assumes that $g\left(\mu_{i}\right)=\operatorname{logit}\left(\mu_{i}\right)=\ln \left(\frac{\mu_{i}}{1-\mu_{i}}\right)=\alpha+\sum_{j=1}^{p} s_{j}\left(x_{i j}\right)$, where $\mu_{i}=p_{i}=E\left(y_{i}=1 \mid x_{i}\right)=\exp \left\{\alpha+\sum_{j=1}^{p} s_{j}\left(x_{i j}\right)\right\} / \exp \left\{1+\alpha+\sum_{j=1}^{p} s_{j}\left(x_{i j}\right)\right\}$.

The adjusted dependent variable $\mathrm{z}$ and the weights $\mathrm{w}$ used in the algorithm above are

$z_{i}=\eta_{i}+\left(y_{i}-p_{i}\right) / p_{i}\left(1-p_{i}\right)$,

$w_{i}=p_{i}\left(1-p_{i}\right)$

where $p_{i}=g^{-1}\left(\eta_{i}\right), \eta_{i}=\alpha+\sum_{j=1}^{p} s_{j}\left(x_{i j}\right)$

The functions $s_{1}, s_{2}, \ldots, s_{p}$ are estimated by an algorithm like the one described earlier.

\subsection{An Empirical Application of GAM Logit Model to data on brand choice of crackers}

The dataset is from Jain et al. [10] and Paap and Franses [13]. We use an optical scanner panel data set on purchases of saltine crackers in the Rome (Georgia) market, collected by Information Resources Incorporated. The data set contains information on all 3292 purchases of crackers of 136 households over a period of two years, including brand choice, actual price of the purchased brand and shelf price of other brands, and whether there was a display and/or newspaper feature of the considered brands at the time of purchase. The data file contains 17 variables, arranged in five rows for each observation as follows.

$I D$ : individual identifiers

CHOICE: one of Sunshine, Keebler, Nabisco, Private Label

$D I S P . z:$ is there a display for brand $\mathrm{z}$ ?

FEAT.z: is there a newspaper feature advertisement for brand $\mathrm{z}$ ?

PRICE.z: price of brand $\mathrm{z}$

1: Household ID 
2-5: Purchase/no-purchase of Sunshine, Keebler, Nabisco, Private Label 6-9: Display/no-display of Sunshine, Keebler, Nabisco, Private Label 10-13: Feature/no-feature of Sunshine, Keebler, Nabisco, Private Label 14-17: Price in \$/unit for Sunshine, Keebler, Nabisco, Private Label

Table 10 shows some data characteristics. There are three major national brands in our database, that is, Sunshine, Keebler and Nabisco with market shares of 7\%, 7\% and 54\%, respectively. The local brands are collected under 'Private label', which has a market share of 32\%; see the first row of Table 10. 'Display' refers to the fraction of purchase occasions that a brand was on display and 'feature' refers to the fraction that a brand was featured. The market leader, Nabisco, is relatively often on display (34\%) and featured (9\%). The 'average price' denotes the mean of the price of a brand over the 3292 purchases. The Keebler crackers seem to be the most expensive crackers in our data set. Table 10 provides information of the number of brand switches in the sample. For example, in $39 \%$ of the cases households buying Sunshine on the current purchase occasion buy the same brand on the next purchase

Table 10: Some data characteristics of the saltine crackers Sunshine, Keebler, Nabisco, and Private label

\begin{tabular}{lllll}
\multicolumn{5}{c}{ Table 10: Some data characteristics of the saltine crackers Sunshine, Keebler, Nabisco, and Private label } \\
\hline BRAND & Sunshine & Keebler & Nabisco & Private Label \\
\hline MKT. SHARE & 0.07 & 0.07 & 0.54 & 0.32 \\
DISPLAY a & 0.13 & 0.11 & 0.34 & 0.10 \\
FEATURE b & 0.04 & 0.04 & 0.09 & 0.05 \\
AVERAGE PRICE & 0.96 & 1.13 & 1.08 & 0.68 \\
ESTIMATED S & 0.39 & 0.07 & 0.35 & 0.19 \\
BRAND K & 0.09 & 0.50 & 0.30 & 0.11 \\
SWITCHING N & 0.04 & 0.04 & 0.84 & 0.08 \\
PROBABILITIES c p & 0.04 & 0.03 & 0.12 & 0.81 \\
\hline
\end{tabular}

The dependent variable $C H O I C E$ is defined as follows.

$N A B I S C O=1$ if Sunshine is chosen,

$=0$ if any other brand is chosen

PRICE.SUNSHINE $=$ Price of a box of Sunshine

PRICE.KEEBLER = Price of a box of Sunshine

PRICE.NABISCO $=$ Price of a box of Sunshine

PRICE.PRIVATE $=$ Price of a box of Sunshine

DISP.SUNSHINE $=1$ if Sunshine is displayed at time of purchase, otherwise $=0$

DISP.KEEBLER $=1$ if Keebler is displayed at time of purchase, otherwise $=0$

DISP.NABISCO $=1$ if Nabisco is displayed at time of purchase, otherwise $=0$

DISP.PRIVATE $=1$ if Private Label is displayed at time of purchase, otherwise $=0$

\section{Models}

The following models were fitted to the data. Models 1 and 2 are Logit models. Given the nonlinearity of the Logit link function in PRATIO as displayed in the partial residual plots of PRICE.NABISCO in Fig.2, Model 3, a Generalized Additive Logit model introduces a nonparametric smooth term s(PRICE.NABISCO). Model 4, another GAM model introduces nonparametric smooth terms $S$ (PRICE.NABISCO), s(PRICE.SUNSHINE) and $s($ PRICE.KEEBLER) in addition to parametric terms for FEAT.NABISCO, FEAT.SUNSHINE, and FEAT.KEEBLER, Finally, Model 5 is a GAM Logit model, which contains no parametric terms, but contains nonparametric smooth terms $s(P R I C E . N A B I S C O)$, s(PRICE.SUNSHINE), s(PRICE.KEEBLER), and s(PRICE.PRIVATE). 
Model 1: Logit Re gression Model 1

$\eta=g(\mu)=\beta_{1}+\beta_{2}$ PRICE.NABISCO $+\beta_{3}$ DISP.NABISCO + $\beta_{4}$ FEAT .NABISCO

Model 2: Logit Re gression Model 2

$\eta=g(\mu)=\beta_{1}+\beta_{2}$ PRICE.KEEBLER $+\beta_{3}$ DISP.KEEBLER $+\beta_{4}$ FEAT .KEEBLER

Model 3:Generalized Additive Logit Re gression Models with a

Nonparametric Interaction Term

$\eta=g(\mu)=\beta_{1}+s($ PRICE.NABISCO $)+\beta_{3}$ DISP.NABISCO + $\beta_{4}$ FEAT .NABISCO

Model 4: Generalized Additive Logit Re gression Models with a Nonparametric

Interaction Term and a dummy variable for whether a particular brand is

featured at the time of purchase

$\eta=g(\mu)=\beta_{1}+\beta_{2}$ FEAT .NABISCO + $\beta_{3}$ FEAT .SUNSHINE + $\beta_{4}$ FEAT .KEEBLER

$+s($ PRICE.NABISCO $)+s($ PRICE.SUNSHINE $)+s($ PRICE.KEEBLER $)$

Model 5: Logit Re gression Model with a Nonparametric Smooth for the

Price of each Brand

$\eta=g(\mu)=\beta_{1}+s($ PRICE.NABISCO $)+s($ PRICE.SUNSHINE $)+s($ PRICE.KEEBLER $)$

$+s($ PRICE.PRIVATE)

Table 11: Model 1: GLM Logit with Nabisco characteristics as predictors: Cracker Data

\begin{tabular}{lllll} 
& \multicolumn{3}{c}{ Table 11: Model 1: GLM Logit with Nabisco characteristics as predictors: Cracker Data } \\
\hline Variable & Coefficient Estimate & Std. Error & t-ratio & p-value \\
\hline Intercept & 2.941133 & 0.290899 & 10.111 & $<2 \times 10^{-16^{* * * *}}$ \\
PRICE.NABISCO & -0.026398 & 0.002626 & -10.051 & $<2 \times 10^{-16^{* * * *}}$ \\
DISP.NABISCO & 0.138151 & 0.076595 & 1.804 & 0.0713 \\
FEAT.NABISCO & 0.588538 & 0.140506 & 4.189 & $2.81 \times 10^{-5 * * *}$ \\
\hline
\end{tabular}

Signif. codes: 0 ‘***’ 0.001 '**' 0.01 '*’ 0.05 '? 0.1 ' ’ 1

(Dispersion parameter for binomial family taken to be 1)

Null deviance: 4537.7 on 3291 degrees of freedom

Residual deviance: 4385.8 on 3288 degrees of freedom

AIC: 4393.8

Number of Fisher Scoring iterations: 4

Table 12: Model 2: GLM Logit with Keebler characteristics as predictors

\begin{tabular}{lllll}
\hline Variable & Coefficient Estimate & Std. Error & t-ratio & p-value \\
\hline Intercept & -0.724835 & 0.383416 & -1.890 & 0.0587 \\
PRICE.KEEBLER & 0.007856 & 0.003369 & 2.332 & $0.0197 *$ \\
DISP.KEEBLER & 0.187321 & 0.122768 & 1.526 & 0.1271 \\
FEAT.KEEBLER & -0.031748 & 0.186839 & -0.170 & 0.8651 \\
\hline
\end{tabular}

Signif. codes: 0 ‘***’ 0.001 '**’ 0.01 '*’ 0.05 ‘' 0.1 “' 1

(Dispersion parameter for binomial family taken to be 1)

Null deviance: 4537.7 on 3291 degrees of freedom

Residual deviance: 4530.7 on 3288 degrees of freedom

AIC: 4538.7

Number of Fisher Scoring iterations: 3 


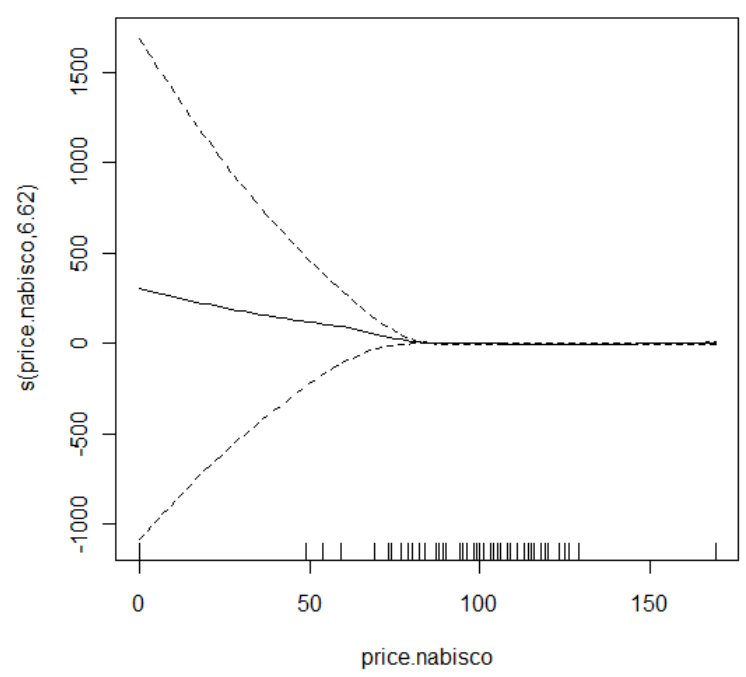

Fig. 2: Partial residuals plot of PRICE.NABISCO

Table 13: Model 3: GAM Logit with a nonparametric smooth term for PRICE.NABISCO

\begin{tabular}{lllll}
\hline Variable & Coefficient Estimate & Std. Error & t-ratio & p-value \\
\hline Intercept & 1.20856 & 1.27053 & 0.951 & 0.3415 \\
DISP.NABISCO & 0.15956 & 0.07876 & 2.026 & $0.0428^{*}$ \\
FEAT.NABISCO & 0.63135 & 0.14305 & 4.414 & $1.02 \times 10^{-5 * * *}$ \\
\hline
\end{tabular}

Approximate significance of smooth terms:

\begin{tabular}{|l|l|l|l|l|}
\hline Variable & Estimated df & Refined df & Chi-squared & p-value \\
\hline$s($ PRICE.NABISCO $)$ & 6.616 & 6.861 & 48.02 & $3.04 \times 10^{-8} * * *$ \\
\hline
\end{tabular}

Signif. codes: 0 ‘***' 0.001 '**’ 0.01 '*’ 0.05 ‘’ 0.1 ' ' 1

R-sq. $(\operatorname{adj})=0.0499$ Deviance explained $=4.53 \%$

UBRE score $=0.32181$ Scale est. $=1 \quad \mathrm{n}=3292$

$\mathrm{AIC}=4351.397$

Null Deviance: 4537.747 on 3291 degrees of freedom

Residual Deviance: 4352.563 on 3285 degrees of freedom

Number of Local Scoring Iterations: 9

Table 14: Model 4: A GAM Logit Model with a nonparametric smooth term for each brand price and a dummy variable for whether a brand is featured at the time of purchase

\begin{tabular}{lllll}
\hline Variable & Coefficient Estimate & Std. Error & t-ratio & p-value \\
\hline Intercept & 67.59466 & 46.81464 & 1.444 & 0.1488 \\
FEAT.SUNSHINE & 0.02945 & 0.21710 & 0.136 & 0.8921 \\
FEAT.NABISCO & 0.30313 & 0.14966 & 2.025 & $0.0428^{*}$ \\
FEAT.KEEBLER & 0.34561 & 0.19080 & 1.811 & 0.0701 \\
\hline
\end{tabular}

Signif. codes: 0 ‘***’ 0.001 '**’ 0.01 '*’ 0.05 ‘’ 0.1 ' 1

Approximate significance of smooth terms:

\begin{tabular}{|l|l|l|l|l|}
\hline Variable & Estimated df & Refined df & Chi-squared & $\mathrm{p}$-value \\
\hline$s($ PRICE.SUNSHINE $)$ & 7.159 & 8.147 & 17.98 & $0.0231^{*}$ \\
\hline$s($ PRICE.NABISCO $)$ & 7.575 & 7.880 & 64.17 & $6.15 \times 10^{-11^{* * * *}}$ \\
\hline$s($ PRICE.KEEBLER $)$ & 8.819 & 8.982 & 121.41 & $<2 \times 10^{-16}$ \\
\hline
\end{tabular}

Signif. codes: 0 ‘***’ 0.001 '**' 0.01 ‘*’ 0.05 ‘’ 0.1 “ ’ 1 
R-sq. $($ adj $)=0.0959$ Deviance explained $=8.43 \%$

UBRE score $=0.279$ Scale est. $=1 \quad n=3292$

$\mathrm{AIC}=4210.49$

Null Deviance: 4537.747 on 3291 degrees of freedom

Residual Deviance: 4265.343 on 3276 degrees of freedom

Number of Local Scoring Iterations: 9

Table 15: Model 5: A GAM Logit Model with a nonparametric smooth term for each brand price

\begin{tabular}{|c|c|c|c|c|}
\hline Variable & Estimated df & Refined df & Chi-squared & $\mathrm{p}$-value \\
\hline s(PRICE.SUNSHIN) & 7.767 & 8.585 & 72.91 & $2.56 \times 10^{-12} *$ \\
\hline$s(P R I C E . N A B I S C O)$ & 7.474 & 7.763 & 88.42 & $7.18 \times 10^{-16^{* * *}}$ \\
\hline$s(P R I C E . K E E B L E R)$ & 8.660 & 8.940 & 95.01 & $<2 \times 10^{-16^{* * *}}$ \\
\hline s(PRICE.PRIVATE $)$ & 8.977 & 9.000 & 386.09 & $<2 \times 10^{-16^{* * *}}$ \\
\hline
\end{tabular}

Parametric Coefficients

\begin{tabular}{|l|l|l|l|l|}
\hline Variable & Coefficient Estimate & Std. Error & t-ratio & p-value \\
\hline Intercept & 88.91 & 45.06 & 1.973 & $0.0485^{*}$ \\
\hline
\end{tabular}

Approximate significance of smooth terms:

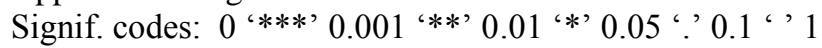

R-sq. $(\operatorname{adj})=0.222$ Deviance explained $=18.7 \%$

UBRE score $=0.14172$ Scale est. $=1 \quad \mathrm{n}=3292$

AIC: 3758.557

Null Deviance: 4537.747 on 3291 degrees of freedom

Residual Deviance: 3945.672 on 3275 degrees of freedom

Number of Local Scoring Iterations: 9

\subsection{Comparing the Models}

Estimation results are presented in tables 11 through 15. A comparison of models using the AIC is presented in Table 16.

\begin{tabular}{ll}
\multicolumn{3}{l}{ Table 16: Models and the AICs } \\
\hline MODEL & AIC \\
\hline 1 & 4393.78 \\
2 & 4538.65 \\
3 & 4351.397 \\
4 & 4210.49 \\
5 & 3758.557 \\
\hline
\end{tabular}

Model 5, a GAM, which includes a nonparametric smooth term for the price of each brand, has the lowest AIC and UBRE score among the five models studied and is therefore the best model. The Logit regression model (Model 2), which uses the characteristics of the competing brand, Keebler has the highest AIC. This is not surprising since the Logit model misses the nonlinearity in the price of each brand in the link function. Model 5 also has the lowest deviance of 3945.672 on 3275 degrees of freedom, while Model 2 has the highest deviance of 4530.7 on 3288 degrees of freedom. The statistical significance of brand prices differs markedly between the Logit regression model and the various GAM Logit models employed here. The signs of the coefficient estimates are all expected in all of the models but Model 2. For instance, the sign of DISP.KEEBLER is positive in model 2 indicating that the odds of choosing NABISCO over KEEBLER increase if KEEBLER is displayed at the time of purchase and decrease if NABISCO is displayed at the time of purchase. The analysis of deviance in Tables 13, 14, and 15 indicates significant nonlinear contribution from the variables PRICE.NABISCO as well as other brand price variables. The high degree of nonlinearity in PRICE.NABISCO is also seen in the partial residual smoothing plot of PRICE.NABISCO in Fig. 2. The dotted curves around the solid curve represent +-2 standard errors around the solid curve. The only surprising result is the negative sign of the variable FEAT.KEEBLER in Table 12. 


\section{The generalized additive Poisson and Negative Binomial Models}

Generalized additive models can be used in virtually any setting where linear models are used. The basic idea is to replace $\sum x_{i j} \beta_{j}$, the linear component of the model with an additive component $\sum f_{j}\left(x_{i j}\right)$.

In the Poisson regression model the outcome, $y_{i}$ is a count variable, such as number of visits to a doctor's office as in Gurmu [5]. We wish to model $p\left(y_{i} \mid x_{i 1}, x_{i 2}, \ldots, x_{i p}\right)$ the probability of an event given factors $x_{i l}, x_{i 2}, \ldots, x_{i p}$. The Poisson regression model assumes that the link function is linear:

$\ln \mu_{i}=\beta_{0}+x_{i 1} \beta_{1}+\ldots+x_{i p} \beta p$

The generalized additive Poisson model assumes instead that

$\ln \mu_{i}=\beta_{0}+s_{1}\left(x_{i 1}\right)+\ldots+s_{p}\left(x_{i p}\right)$

The functions $s_{1}, s_{2}, \ldots, s_{p}$ are estimated by maximizing a penalized log likelihood or a penalized log partial-likelihood using the PIRLS algorithm described above.

\subsection{An Empirical Application of GAM Poisson and GAM Negative Binomial Models to data on doctor visits}

\section{Data and variable definitions}

The data are a 1986 cross section sample from the US consisting of 485 observations and are drawn from Gurmu [5]. These data came from the 1986 Medicaid Consumer Survey sponsored by the Health Care Financing Administration. The following variables were used in econometric analysis.

$D O C T O R=$ the number of doctor visits

CHILDREN = the number of children in the household

ACCESS $=$ is a measure of access to health care

$H E A L T H=$ a measure of health status (larger positive numbers are associated with poorer health

\begin{tabular}{|c|c|c|c|c|c|}
\hline Variable & Obs. & Mean & Std. Dev. & Min & Max \\
\hline DOCTOR & 485 & 1.610 & 3.346809 & 0 & 48 \\
\hline CHILDREN & 485 & 2.264 & 1.319136 & 1 & 9 \\
\hline ACCESS & 485 & 0.3812 & 0.186105 & 0 & 0.92 \\
\hline HEALTH & 485 & -0.00004124 & 1.433520 & 1 & 1 \\
\hline
\end{tabular}

A likelihood ratio test of $\mathrm{H}_{0}$ : Poisson against $\mathrm{H}_{1}$ : NB model yielded a Chi-Square Test Statistic $=599.6065$ and p-value $=<2.2 \mathrm{e}-16$ and overdispersion was confirmed. Accordingly, both Poisson and Negative Binomial GLM and GAM were fitted to the data.

\section{Models}

The following models were fitted to the data. Models $1 \mathrm{P}$ and $1 \mathrm{NB}$ are Poisson and Negative Binomial regression models, which use CHILDREN, ACCESS and HEALTH as independent variables. Given the nonlinearity of the Logit link function in HEALTH as displayed in the partial residual plots of HEALTH in Fig. 3, Models $2 \mathrm{P}$ and $2 \mathrm{NB}$ are Generalized Additive Poisson and Negative Binomial models respectively, which introduce a nonparametric smooth term $s(H E A L T H)$. Models 3P and 3NB are Generalized Additive Poisson and Negative Binomial models respectively, which introduce nonparametric smooth terms $s$ (ACCESS) and $s(H E A L T H)$. Models $4 \mathrm{P}$ and $4 \mathrm{NB}$ are Generalized Additive Poisson and Negative Binomial models, which introduce a nonparametric smooth interaction term $s\left(A C C E S S^{*} H E A L T H\right)$ in addition to nonparametric smooth terms $s(A C C E S S)$ and $s(H E A L T H)$. Models $5 \mathrm{P}$ and $5 \mathrm{NB}$ replace the nonparametric interaction $s\left(A C C E S S^{*} H E A L T H\right)$ with another nonparametric interaction $s\left(A C C E S S^{*} C H I L D R E N\right)$. Additional GAM models with more general nonparametric interaction terms $s(A C C E S S, H E A L T H)$ and $s(A C C E S S, C H I L D R E N)$ were also estimated but did not result in improvements and are not reported here. 
Model 1P: Poisson Re gression Model with Identity Link

$\eta=g(\mu)=\beta_{1}+\beta_{2}$ CHILDREN $+\beta_{3}$ ACCESS $+\beta_{4}$ HEALTH

Model $1 N B:$ Negative Binomial Re gression with Identity Link

$\eta=g(\mu)=\beta_{1}+\beta_{2}$ CHILDREN $+\beta_{3}$ ACCESS $+\beta_{4}$ HEALTH

Model 2P:Generalized Additive Poisson Re gression Model

$\eta=g(\mu)=\beta_{1}+\beta_{2} C H I L D R E N+\beta_{3} A C C E S S+s(H E A L T H)$

Model 2NB: Generalized Additive Negative Binomial Re gression Model

$\eta=g(\mu)=\beta_{1}+\beta_{2}$ CHILDREN $+\beta_{3}$ ACCESS $+s($ HEALTH $)$

Model 3P: Generalized Additive Poisson Re gression Model with smooth

nonparametric terms for access and health

$\eta=g(\mu)=\beta_{1}+\beta_{2} C H I L D R E N+s($ ACCESS $)+s(H E A L T H)$

Model 3NB:Generalized Additive Negative Binomial Re gression Model with smooth nonparametric terms for access and health

$\eta=g(\mu)=\beta_{1}+\beta_{2} C H I L D R E N+s($ ACCESS $)+s($ HEALTH $)$

Model 4P: Generalized Additive Poisson Re gression Model with smooth

nonparametric terms for access and health and nonparametric int eraction between

ACCESS and HEALTH

$\eta=g(\mu)=\beta_{1}+\beta_{2}$ CHILDREN $+s($ ACCESS $)+s($ HEALTH $)+s($ ACCESS * HEALTH $)$

Model 4NB: Generalized Additive Negative Binomial Re gression Model with smooth nonparametric terms for access and health and nonparametric int eraction between

ACCESS and HEALTH

$\eta=g(\mu)=\beta_{1}+\beta_{2}$ CHILDREN $+s($ ACCESS $)+s($ HEALTH $)+s($ ACCESS * HEALTH $)$

Model 5P: Generalized Additive Poisson Re gression Model with smooth

nonparametric terms for access and health and nonparametric int eraction between

ACCESS and CHILDREN

$\eta=g(\mu)=\beta_{1}+\beta_{2}$ CHILDREN $+s($ ACCESS $)+s($ HEALTH $)+s($ ACCESS * CHILDREN $)$

Model 5NB: Generalized Additive Negative Binomial Re gression Model with smooth nonparametric terms for access and health and nonparametric int eraction between

ACCESS and CHILDREN

$\eta=g(\mu)=\beta_{1}+\beta_{2}$ CHILDREN $+s($ ACCESS $)+s($ HEALTH $)+s($ ACCESS * CHILDREN $)$

Table 18: Model 1P: Poisson Regression Model

\begin{tabular}{lllll}
\hline Variable & Coefficient Estimate & Std. Error & t-ratio & p-value \\
\hline INTERCEPT & 0.37509 & 0.11016 & 3.405 & $0.000662^{* * *}$ \\
CHILDREN & -0.17592 & 0.03164 & -5.560 & $2.70 \times 10^{-8}$ \\
ACCESS & 0.93694 & 0.19278 & 4.860 & $1.17 \times 10^{-6}$ \\
HEALTH & 0.28984 & 0.01825 & 15.880 & $<2 \times 10^{-16}$ \\
\hline
\end{tabular}

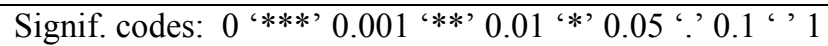

(Dispersion parameter for poisson family taken to be 1)

Null deviance: 1766.2 on 484 degrees of freedom

Residual deviance: 1508.8 on 481 degrees of freedom

AIC: 2179.5

Number of Fisher Scoring iterations: 6

Table 19: Model 1NB: Negative Binomial Regression Model

\begin{tabular}{lllll}
\hline Variable & Coefficient Estimate & Std. Error & t-ratio & $\mathrm{p}$-value \\
\hline INTERCEPT & 0.48207 & 0.24005 & 2.008 & $0.0452^{*}$ \\
CHILDREN & -0.16573 & 0.06744 & -2.457 & $0.0144^{*}$ \\
ACCESS & 0.60193 & 0.43715 & 1.377 & 0.1692 \\
HEALTH & 0.30344 & 0.04990 & 6.081 & $2.43 \times 10^{-9 * * *}$ \\
\hline
\end{tabular}

Signif. codes: 0 ‘***’ 0.001 '**’ 0.01 ‘*’ 0.05 '? $0.1^{\text {‘ ’ } 1}$ 
(Dispersion parameter for Negative Binomial(2) family taken to be 2.653272)

Null deviance: 940.44 on 484 degrees of freedom

Residual deviance: 813.49 on 481 degrees of freedom

AIC: 1692.6

Number of Fisher Scoring iterations: 5

Table 20: Model 2P: Generalized Additive Poisson Regression Model

\begin{tabular}{lllll}
\hline Variable & Coefficient Estimate & Std. Error & t-ratio & p-value \\
\hline INTERCEPT & 0.3182 & 0.1140 & 2.792 & $0.00523^{* * *}$ \\
CHILDREN & -0.1772 & 0.0319 & -5.555 & $2.77 \times 10^{-8 * * *}$ \\
ACCESS & 0.9933 & 0.1990 & 4.991 & $6.02 \times 10^{-7^{* * *}}$ \\
\hline
\end{tabular}

Family: Poisson

Link function: $\log$

Approximate significance of smooth terms:

\begin{tabular}{|l|l|l|l|l|}
\hline Variable & Estimated df & Refined df & Ch-squared & p-value \\
\hline$s($ HEALTH $)$ & 8.62 & 8.952 & 300.5 & $<2 \times 10^{-16^{* * *}}$ \\
\hline
\end{tabular}

Signif. codes: 0 ‘***’ 0.001 '**’ 0.01 ‘*’ 0.05 ‘’ 0.1 ' ' 1

R-sq. $(\operatorname{adj})=0.142$ Deviance explained $=17.6 \%$

UBRE score $=2.0474$ Scale est. $=1 \quad n=485$

AIC: 2140.685

Null Deviance: 1766.246 on 484 degrees of freedom

Residual Deviance: 1443.896 on 475.0002 degrees of freedom

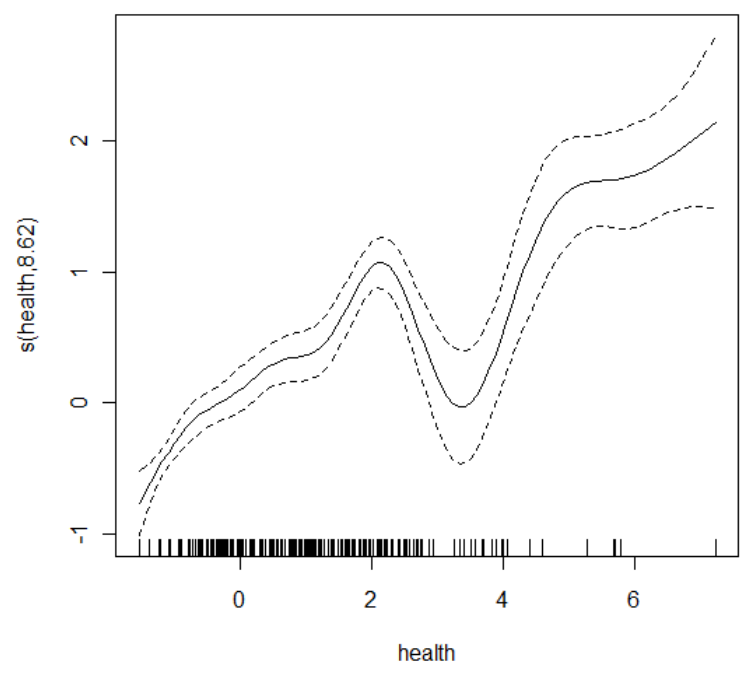

Fig. 3: Partial Residual plot of HEALTH

Table 21: Model 2NP: GAM Negative Binomial (2) Regression Model

\begin{tabular}{lllll}
\hline Variable & Coefficient Estimate & Std. Error & t-ratio & p-value \\
\hline INTERCEPT & 0.50987 & 0.14996 & 3.400 & $0.000674^{* * * *}$ \\
CHILDREN & -0.17116 & 0.04199 & -4.076 & $4.58 \times 10^{-5 * * *}$ \\
ACCESS & 0.48808 & 0.27428 & 1.779 & 0.075162 \\
\hline
\end{tabular}

Family: Negative Binomial (2)

Link function: $\log$

Approximate significance of smooth terms: 


\begin{tabular}{|l|l|l|l|l|}
\hline Variable & Estimated df & Refined df & Ch-squared & p-value \\
\hline$s($ HEALTH $)$ & 8.268 & 8.836 & 105.9 & $<2 \times 10^{-16^{* * * *}}$ \\
\hline
\end{tabular}

Signif. codes: 0 ‘***’ 0.001 '**’ 0.01 ‘*’ 0.05 ‘’ $0.1^{\prime}$ ' 1

R-sq. $(\operatorname{adj})=0.115$ Deviance explained $=15.7 \%$

UBRE score $=0.68084$ Scale est. $=1 \quad \mathrm{n}=485$

AIC: 1686.335

Null Deviance: 1766.246 on 484 degrees of freedom

Residual Deviance: 1478.552 on 477.9999 degrees of freedom

Table 22: Model 3P: GAM Poisson Regression Model with nonparametric smooth terms for ACCESS and HEALTH

\begin{tabular}{lllll}
\hline Variable & Coefficient Estimate & Std. Error & t-ratio & p-value \\
\hline INTERCEPT & 0.62717 & 0.07672 & 8.175 & $2.97 \times 10^{-16^{* * *}}$ \\
CHILDREN & -0.16883 & 0.03165 & -5.334 & $9.63 \times 10^{-8^{* * *}}$ \\
\hline
\end{tabular}

Approximate significance of smooth terms:

\begin{tabular}{|l|l|l|l|l|}
\hline Variable & Estimated df & Refined df & Ch-squared & p-value \\
\hline$s($ ACCESS $)$ & 8.62 & 8.962 & 300.5 & $<2 \times 10^{-16^{* * * *}}$ \\
\hline$S($ HEALTH $)$ & 8.437 & 8.902 & 238.4 & $<2 \times 10^{-16^{* * * *}}$ \\
\hline
\end{tabular}

Signif. codes: 0 ‘***' 0.001 '**' 0.01 '*’ 0.05 '? $0.1^{\prime}$ ' 1

R-sq. $(\operatorname{adj})=0.175$ Deviance explained $=22.7 \%$

UBRE score $=1.8955$ Scale est. $=1 \quad \mathrm{n}=485$

AIC: 2067.047 Family: Poisson

Link function: $\log$

Null Deviance: 1766.246 on 484 degrees of freedom

Residual Deviance: 1443.896 on 475.0002 degrees of freedom

Table 23: Model 3NB: GAM Negative Binomial Regression Model with nonparametric smooth terms for ACCESS and HEALTH

\begin{tabular}{lllll}
\hline Variable & Coefficient Estimate & Std. Error & t-ratio & p-value \\
\hline INTERCEPT & 0.62302 & 0.10382 & 6.001 & $1.96 \times 10^{-9 * * *}$ \\
CHILDREN & -0.16414 & 0.04218 & -3.891 & $9.97 \times 10^{-5 * * *}$ \\
\hline
\end{tabular}

Approximate significance of smooth terms:

\begin{tabular}{|l|l|l|l|l|}
\hline Variable & Estimated df & Refined df & Ch-squared & p-value \\
\hline$s($ ACCESS $)$ & 8.861 & 8.991 & 48.14 & $2.39 \times 10^{-7 * * *}$ \\
\hline$S($ HEALTH $)$ & 8.041 & 8.734 & 94.46 & $<2 \times 10^{-16^{* * *}}$ \\
\hline
\end{tabular}

Signif. codes: 0 '***' 0.001 '**' 0.01 '*’ 0.05 '? 0.1 ' ' 1

R-sq. $(\operatorname{adj})=0.137$ Deviance explained $=20.8 \%$

UBRE score $=0.61309$ Scale est. $=1 \quad n=485$

AIC: 1653.477

Table 24: Model 4P: GAM Poisson Regression Model

\begin{tabular}{lllll}
\hline Variable & Coefficient Estimate & Std. Error & t-ratio & p-value \\
\hline INTERCEPT & 0.60408 & 0.07785 & 7.759 & $8.54 \times 10^{-15^{* * * *}}$ \\
CHILDREN & -0.16390 & 0.03206 & -5.113 & $3,17 \times 10^{-7^{* * * *}}$ \\
\hline
\end{tabular}

Approximate significance of smooth terms:

\begin{tabular}{|l|l|l|l|l|}
\hline Variable & Estimated df & Refined df & Ch-squared & p-value \\
\hline$s($ ACCESS $)$ & 8.790 & 8.978 & 79.14 & $2.34 \times 10^{-13^{* * *}}$ \\
\hline$S($ HEALTH $)$ & 8.099 & 8.730 & 33.51 & $8.86 \times 10^{-5^{* * * *}}$ \\
\hline$s\left(\right.$ ACCESS $\left.{ }^{*} H E A L T H\right)$ & 5.427 & 6.630 & 36.31 & $4.47 \times 10^{-6^{* * *}}$ \\
\hline
\end{tabular}

Signif. codes: 0 ‘***’ 0.001 '**’ 0.01 '*’ 0.05 '? 0.1 ' ' 1

R-sq. $(\operatorname{adj})=0.232$ Deviance explained $=25.6 \%$ 
UBRE score $=1.8088$ Scale est. $=1 \quad \mathrm{n}=485$

AIC: 2024.952

Null Deviance: 1766.246 on 484 degrees of freedom

Residual Deviance: 1380.621 on 471.0001 degrees of freedom

Table 25: Model 4NB: GAM Negative Binomial Regression Model with Nonparametric Interaction

\begin{tabular}{lllll}
\hline Variable & Coefficient Estimate & Std. Error & t-ratio & p-value \\
\hline INTERCEPT & 0.60298 & 0.10415 & 5.790 & $7.05 \times 10^{-9 * * * *}$ \\
CHILDREN & -0.15887 & 0.04227 & -3.759 & $0.000171^{* * *}$ \\
\hline
\end{tabular}

Approximate significance of smooth terms:

\begin{tabular}{|l|l|l|l|l|}
\hline Variable & Estimated df & Refined df & Ch-squared & p-value \\
\hline$s($ ACCESS $)$ & 8.717 & 8.966 & 50.110 & $9.95 \times 10^{-8 * * *}$ \\
\hline$S(H E A L T H)$ & 1.263 & 1.467 & 1.555 & 0.3289 \\
\hline$s(\text { ACCESS } \text { HEALTH })^{*}$ HEA & 4.465 & 5.548 & 18.901 & $0.0031^{* *}$ \\
\hline
\end{tabular}

Signif. codes: 0 ‘***' 0.001 '**' 0.01 '*’ 0.05 '? 0.1 ' ' 1

R-sq. $(\operatorname{adj})=0.15$ Deviance explained $=21.6 \%$

UBRE score $=0.58768$ Scale est. $=1 \quad \mathrm{n}=485$

AIC: 1641.151

Table 26: Model 5P: GAM Poisson Regression Model with Nonparametric interaction

\begin{tabular}{|c|c|c|c|c|}
\hline Variable & Coefficient Estimate & Std. Error & t-ratio & p-value \\
\hline INTERCEPT & 0.10745 & 0.18992 & 0.566 & 0.572 \\
\hline CHILDREN & 0.05892 & 0.08160 & 0.722 & 0.470 \\
\hline
\end{tabular}

Approximate significance of smooth terms:

\begin{tabular}{|l|l|l|l|l|}
\hline Variable & Estimated df & Refined df & Ch-squared & p-value \\
\hline$s($ ACCESS $)$ & 8.921 & 8.997 & 116.010 & $<2 \times 10^{-16^{* * *}}$ \\
\hline$S($ HEALTH $)$ & 8.568 & 8.940 & 243.771 & $<2 \times 10^{-16^{* * *}}$ \\
\hline$s\left(\right.$ ACCESS ${ }^{*}$ CHILDREN $)$ & 1.341 & 1.625 & 8.819 & $0.00772^{* * *}$ \\
\hline
\end{tabular}

Signif. codes: 0 '***' 0.001 '**' 0.01 '*' 0.05 ' ' 0.1 ' ' 1

R-sq. $(\operatorname{adj})=0.188$ Deviance explained $=23.2 \%$

UBRE score $=1.8812$ Scale est. $=1 \quad n=485$

AIC: 2060.085

Null Deviance: 1766.246 on 484 degrees of freedom

Residual Deviance: 1426.358 on 471 degrees of freedom

Table 27: Model 5NB: GAM Negative Binomial Regression Model with Nonparametric Interaction

\begin{tabular}{lllll}
\hline Variable & Coefficient Estimate & Std. Error & t-ratio & p-value \\
\hline INTERCEPT & 0.33961 & 0.24609 & 1.380 & 0.168 \\
CHILDREN & -0.03982 & 0.10631 & -0.375 & 0.708 \\
\hline
\end{tabular}

Approximate significance of smooth terms:

\begin{tabular}{|l|l|l|l|l|}
\hline Variable & Estimated df & Refined df & Ch-squared & p-value \\
\hline$s($ ACCESS $)$ & 8.862 & 8.992 & 49.488 & $1.33 \times 10^{-7 * * *}$ \\
\hline$S($ HEALTH $)$ & 8.175 & 8.797 & 94.602 & $<2 \times 10^{-16^{* * *}}$ \\
\hline$s\left(\right.$ ACCESS ${ }^{*}$ CHILDREN $)$ & 1.000 & 1.001 & 1.527 & 0.217 \\
\hline
\end{tabular}

Signif. codes: 0 ‘***’ 0.001 '**’ 0.01 '*’ 0.05 ‘' 0.1 “ ' 1

R-sq. $(\operatorname{adj})=0.144$ Deviance explained $=21 \%$

UBRE score $=0.61398$ Scale est. $=1 \quad \mathrm{n}=485$

AIC: 1653.908 


\subsection{Comparing the models}

Estimation results are presented in tables 18 through 27. A comparison of models using the AIC is presented in Tables 28 and 29. Table 28 compares GLM and GAM Poisson models and Table 29 compares GLM and GAM Negative Binomial models. Model 4P, a GAM Poisson model, which includes nonparametric smooth terms for ACCESS, HEALTH and the interaction ACCESS ${ }^{*} H E A L T H$ has the lowest AIC and UBRE score among the five models studied and is therefore the best model. The GLM Poisson regression model (Model 1P), which uses the predictors CHILDREN, ACCESS and HEALTH has the highest AIC. This is not surprising since the Poisson model misses the nonlinearity in the predictors CHILDREN, ACCESS, HEALTH, and interaction ACCESS*HEALTH. Similarly, Model 4NB, a GAM Negative Binomial model, which includes nonparametric smooth terms for ACCESS, HEALTH and the interaction ACCESS $* H E A L T H$ has the lowest AIC and UBRE score among the five models studied and is therefore the best model among the Negative Binomial GLM and GAM models. Finally, among the Poisson and Negative Binomial GLM and GAM models studied here, the clear winner is Model 4NB, which has the lowest AIC and UBRE scores. Model 4P also has the lowest deviance of 1380.621 on 471.0001 degrees of freedom among the Poisson GLM and GAM models, while Model 1P has the highest deviance of 1508.8 on 481 degrees of freedom. The statistical significance of the predictors CHILDREN, ACCESS and HEALTH are generally similar among all models since all of the variables are found highly significant in all of the models. The signs of the coefficient estimates are all expected in all of the models but Model 2. For instance, the sign of ACCESS is positive as is the sign of HEALTH, but the sign of CHILDREN is negative indicating that as access to health services increases, individuals make more trips to a doctor's office even for routine checkups. Individuals with poor health with higher numbers on HEALTH tend to make more trips to a doctor's office as reflected in the positive sign of HEALTH. The negative sign of the variable CHILDREN may be surprising, but may indicate that households with fewer children make more trips to a doctor's office perhaps because these households in the sample are more health-conscious than households with too many children in this sample. The analysis of deviance in Tables 21 through 27 indicates significant nonlinear contribution from the variables ACCESS, HEALTH, and interaction ACCESS*HEALTH. The high degree of nonlinearity in HEALTH is also seen in the partial residual smoothing plot of HEALTH in Figure 3. The dotted curves around the solid curve represent +-2 standard errors around the solid curve.

Table 28: GLM and GAM Poisson Models and the AICs

\begin{tabular}{ll}
\hline MODEL & AIC \\
\hline $1 \mathrm{P}$ & 2179.487 \\
$2 \mathrm{P}$ & 2140,685 \\
$3 \mathrm{P}$ & 2067.047 \\
$4 \mathrm{P}$ & 2024.952 \\
$5 \mathrm{P}$ & 2060.085 \\
\hline
\end{tabular}

Table 29: GLM and GAM Negative Binomial Models and the AICs

\begin{tabular}{ll}
\hline MODEL & AIC \\
\hline 1NB & 1692.614 \\
2NB & 1686.335 \\
3NB & 1653.477 \\
$4 \mathrm{NB}$ & 1641.151 \\
5NB & 1653.908 \\
\hline
\end{tabular}

\section{Conclusion}

The paper has presented econometric applications of generalized additive Gaussian, Logit, Poisson, and Negative Binomial regression models as alternatives to the generalized linear Normal, Logit, Poisson, and Negative Binomial regression models respectively. The Gaussian generalized additive models (GAMs) were applied to data on wages, education, and experience. The Logit GAMS were applied to data on brand choice. The Poisson and Negative Binomial GAMs were applied to data on doctor visits. In all of the empirical applications, each of the GAMs provides a much better fit than the corresponding generalized linear model (GLM) as reflected in lower AICs and lower deviances. The econometric techniques used in the paper are widely applicable to the analysis of count, binary response and duration types of data occurring frequently in economics and business. GAMs extend nonparametric regression to more than one predictor helping to circumvent the curse of dimensionality. Another advantage of the GAM approach used in the paper is that we take account of nonlinearities in predictors and interactions among predictors non-parametrically. Nevertheless, the GAMs are not without drawbacks. The computational algorithms are complex and interpretations can 
be difficult. These models are useful mainly when simple models for the linear predictor provide an inadequate fit for the data.

\section{References}

[1] Baltas, G., Determinants of store brand choice: a behavioral analysis. Journal of Product \& Brand Management, Vol. 6, No.5, (1997), pp. 315 -324 .

[2] Cameron, A. C. and P. Trivedi, Microeconometrics. Cambridge University Press, New York, (1998).

[3] Greene, W. H., Econometric Analysis. Pearson/Prentice Hall, New York, (2008).

[4] Guadagni, P. M. and J. D. Little, A Logit Model of Brand Choice Calibrated on Scanner Data, Marketing Science, Vol. 2, No. 3, (1983), pp. 203-238.

[5] Gurmu, S., Semiparametric estimation of hurdle regression models with an application to medicaid utilization. Journal of Applied Econometrics, Vol. 12, No. 3, (1997), pp. 225-242.

[6] Hastie, T. \& Tibshirani, R., Generalized Additive Models, Statistical Science, Vol. 1, No. 3, (1986), pp. 297-318.

[7] Hastie, T. \& Tibshirani, R., Generalized Additive Models: Some Applications, Journal of the American Statistical Association, Vol. 82, No. 398, (1987), pp. 371-386.

[8] Hastie, T. \& Tibshirani, R., Generalized Additive Models. Chapman and Hall, London, (1990).

[9] Hill, R. C., W. E. Griffiths, and G. C. Lim, Principles of Econometrics, Wiley, New Jersey, (2012).

[10] Jain, D. C., N. J. Vilcassim and P. K. Chintagunta, A random-coefficients logit brand-choice model applied to panel data", Journal of Business and Economics Statistics, Vol. 12, No. 3. (1994), pp. 317-328.

[11] McCullagh, P. and J. Nelder, Generalized Linear Models, Chapman and Hall, London (1989).

[12] Manski, C. and D. McFadden, Structural Analysis of Discrete Data and Econometric Applications, MIT Press, Cambridge, (1981).

[13] Paap, R. and P. H Frances, A dynamic multinomial probit model for brand choices with different short-run effects of marketing mix variables", Journal of Applied Econometrics, Vol. 15, No. 6, (2000), pp. 717-744.

[14] Wood, S. N., R-package mgcv, (2012).

[15] Wood, S.N., Generalized Additive Models: an introduction with R, CRC, Boca Raton, (2006). 